\title{
O impacto da obesidade abdominal sobre os níveis plasmáticos de lípides nos idosos
}

\section{The impact of abdominal obesity on the plasmatic lipid levels in the older people}

Daniela Tiemi Nagatsuyu ${ }^{1}$, Eny K. Uemura Moriguti ${ }^{2}$, Karina Pfrimer ${ }^{3}$, Paulo Fernandes Formighieri ${ }^{3}$, Nereida Kilza da Costa Lima ${ }^{4}$, Eduardo Ferriolli ${ }^{4}$, Julio César Moriguti ${ }^{4}$

\begin{abstract}
RESUMO
Modelo do Estudo: estudo de prevalência. Introdução: Há relatos do aumento da prevalência de dislipidemias com o envelhecimento. Há poucos estudos associando a obesidade centrípeta com a dislipidemia e há, ainda, que se determinar a real influência da obesidade abdominal sobre os níveis plasmáticos de lípides em idosos. Objetivo: Avaliar a possível associação entre a medida da circunferência abdominal, marcadora de gordura visceral, e os níveis de lípides no sangue. Métodos: O estudo foi realizado em 98 pacientes com 60 a 79 anos de idade, moradores do Distrito Oeste da cidade de Ribeirão Preto, sendo que 58 eram mulheres. A idade média dos voluntários foi de 66,3 anos. Foram colhidos os seguintes dados: circunferência abdominal, circunferência do quadril, triglicérides, colesterol total e HDL colesterol. A circunferência abdominal foi o parâmetro para a obesidade centrípeta. Resultados: Não houve associação entre colesterol total e circunferência abdominal $(p=0,88)$. Quando separamos pelo gênero verificamos que também não houve associação $(p=0,73$ e $p=0,60$ para homens e mulheres, respectivamente). A associação entre triglicérides e circunferência abdominal foi significativa $(p<0,0001)$, mas ao separar por gêneros, houve associação entre triglicérides e circunferência abdominal nas mulheres $(p=0,002)$ enquanto que nos homens não houve associação $(p=0,07)$. Houve associação negativa entre HDL e circunferência abdominal $(p=0,018)$, porém quando os gêneros foram analisados separadamente a associação entre HDL e circunferência abdominal não foi significativa ( $p=0,40$ e $p=0,07$ para homens e mulheres, respectivamente). Conclusão: Provavelmente, em idosos, o risco cardiovascular advindo da obesidade centrípeta não tem na sua etiologia, exclusivamente, o aumento de lípides do sangue.
\end{abstract}

Palavras-chave: Colesterol. Circunferência Abdominal. Obesidade. Triglicérides. Idoso.

\section{Introdução}

Envelhecimento é o processo dinâmico no qual as alterações morfológicas, bioquímicas e funcionais tornam o organismo mais suscetível às agressões, que culminam com a morte. ${ }^{1}$ No Brasil, a população de idosos aumentou de aproximadamente 7,2 milhões, em 1980, para 12,6 milhões de idosos em 2000, e esti-

1. Aluna de Iniciação Científica (Bolsista PIBIC), Graduação em Medicina - FMRP-USP;

2. Nutricionista do HCFMRP-USP;

3. Pós-graduando do Departamento de Clínica Médica da FMRP-USP;

4. Docente da Divisão de Clínica Médica Geral e Geriatria da FMRP-USP.

Correspondência: Julio César Moriguti Departamento de Clínica Médica da FMRP-USP Avenida Bandeirantes 3900, 14049-900, Ribeirão Preto - SP e-mail: moriguti@fmrp.usp.br

Artigo recebido em 08/08/2008 Aprovado em 11/03/2009 
ma-se que em 2050 esse número seja 64 milhões. Uma das explicações para esse fato é a de que a expectativa de vida ao nascer teve um aumento de 63,4 anos, em 1980, para 70,4 anos em 2000. ${ }^{2}$

A prevalência de pessoas com sobrepeso e com obesidade também está aumentando na população. A International Obesity Task Force publicou no relatório da Organização Mundial de Saúde a existência, no mundo, de cerca de 250 milhões de adultos obesos (Índice de Massa Corporal - IMC ${ }^{3} 30 \mathrm{Kg} / \mathrm{m}^{2}$ ) e de 500 milhões de indivíduos com sobrepeso (IMC ${ }^{3} 25 \mathrm{Kg}$ / $\left.\mathrm{m}^{2}\right){ }^{3}$ E esse aumento pode refletir na população idosa, uma vez que em estudo realizado em chineses foi identificado que os percentis do IMC aumentam com a idade, tendo o seu pico entre 50 e 59 anos. $^{4}$

Há que se destacar que dentre as alterações que ocorrem com a composição corporal com o envelhecimento, as alterações da massa gordurosa e da massa muscular e óssea são as mais expressivas. É evidente um incremento do tecido gorduroso em detrimento da massa muscular. Esse aumento da massa gordurosa, tanto percentual quanto absoluto, é mais pronunciado no abdome (obesidade abdominal), especialmente em vísceras.

$\mathrm{Na}$ literatura científica, existem inúmeros trabalhos que mostram evidências do aumento do risco cardiovascular relacionado à obesidade abdominal, como o estudo realizado na China com 309 pacientes obesos, que concluiu que o risco cardiovascular relaciona-se especialmente com a obesidade regional, mais especificamente com a obesidade abdominal. ${ }^{5,6}$

Akahoshi e colaboradores, em 2001, estudaram na população idosa de Nagasaki, Japão, a relação da gordura hepática, que é grande marcadora de obesidade visceral, com fatores de risco para doença aterosclerótica, em ambos os gêneros. A gordura hepática foi significativamente relacionada à hipercolesterolemia e hipertrigliceridemia em homens enquanto a hipertensão arterial, a hipercolesterolemia, os baixos níveis de HDL-colesterol (High Density Lipoprotein), a hipertrigliceridemia e o diabetes mellitus ou intolerância a glicose foram em mulheres. ${ }^{7}$

Há relatos do aumento da prevalência de dislipidemias com o envelhecimento. ${ }^{8,9}$ Associado a isto, também está aumentada a prevalência de idosos com obesidade centrípeta, avaliados por meio da obesidade visceral. ${ }^{7}$ Há também estudos mostrando o aumento da relação cintura quadril e da circunferência abdominal com o envelhecimento. ${ }^{6}$ São poucos os estudos que associam a obesidade abdominal com a presença de dislipidemia e há, ainda, que se determinar a real influência da obesidade abdominal sobre os níveis plasmáticos de lípides em idosos.

Outros estudos também mostraram que há relação entre a circunferência abdominal e triglicérides (TG), VLDL-TG (Very Low Density Lipoprotein) e VLDL-colesterol. ${ }^{10,11}$

Na velhice, a obesidade está associada ao aumento da mortalidade e dos riscos de diabetes mellitus tipo 2, intolerância a glicose, aterosclerose, hipertensão arterial, dislipidemia, acidente vascular cerebral, coronariopatias, colelitíase, osteoartrose, alguns tipos de câncer, declínio funcional e invalidez. Idosos obesos têm qualidade de vida pior que a dos não obesos, o que é atribuído ao estilo de vida não saudável e doenças crônicas causadas pela obesidade. ${ }^{4,12,13}$

Todos esses fatores de risco estão interligados, pois a obesidade é uma desordem multifatorial complexa crônica que causa complicações metabólicas, resistência à insulina, diabetes mellitus tipo 2, falência das células das ilhotas de Langerhans, hipertensão arterial e dislipidemia, além de complicações endócrinas (eg, síndrome dos ovários policísticos e superprodução de andrógenos), complicações mecânicas (eg, doenças articulares degenerativas das extremidades inferiores), apnéia obstrutiva do sono (colapso das vias aéreas superiores na inspiração), câncer (eg, de mama, endométrio, próstata e cólon) e distúrbios gastrointestinais (eg, doença do refluxo gastroesofágico e litíase biliar).

O aumento dos níveis de colesterol é explicado pelas complicações metabólicas, nas quais ocorre desrregulação do processo de lipólise, resultando em maior liberação de ácidos graxos e glicerol. Os ácidos graxos livres fornecem a maior parte do combustível lipídico circulante, e seu excesso constitui a hiperlipidemia. ${ }^{14}$ $\mathrm{O}$ tecido adiposo é o maior reservatório de colesterol do organismo. Contém 0,6 a 1,6 mg de colesterol por grama de peso líquido do tecido adiposo. E essa quantidade aumenta com a idade e com o peso. ${ }^{15}$

Dislipidemia e obesidade são muito freqüentes, principalmente nos idosos. Esses dados são preocupantes porque ambos são potenciais fatores de risco para várias doenças, com destaque para a doença aterosclerótica. Assim, necessitam de maior atenção por parte dos pacientes e dos sistemas de saúde.

\section{Objetivos}

O objetivo desse estudo foi avaliar a possível associação entre a medida da circunferência abdominal, que assume-se ser marcadora de gordura visceral, e os níveis de lípides no sangue. 


\section{Casuística e métodos}

O estudo foi realizado em 101 pacientes com 60 a 79 anos de idade, sendo 62 mulheres, sem uso de hipolipemiantes. Todos eram moradores do Distrito Oeste da cidade de Ribeirão Preto, cadastrados nos Programas de Saúde da Família do Distrito em questão. A idade média dos voluntários foi de $65,7 \pm 4,1$ anos (sendo que a dos homens foi de 65,9 anos e a das mulheres foi de 65,6 anos). O Índice de Massa Corpórea foi de $27,64 \pm 4,38 \mathrm{~kg} / \mathrm{m}^{2}$. Foram colhidos os seguintes dados: circunferência abdominal (CA, em centímetros), circunferência do quadril (CQ, em centímetros), triglicérides ( $\mathrm{mg} / \mathrm{dL})$, colesterol total ( $\mathrm{mg} / \mathrm{dL}$ ) e HDL colesterol (mg/dL).

A circunferência abdominal (medida na altura da cicatriz umbilical) foi o parâmetro para a obesidade abdominal. Quando se encontrou deslocamento da cicatriz umbilical, optou-se por medir a circunferência no ponto da metade da distância entre a face inferior da última costela e a porção superior da crista ilíaca. A medida da circunferência do quadril foi tomada pelo ponto de maior circunferência sobre a região glútea, com a fita mantida em plano horizontal, sem pressionar os tecidos moles.

Os exames bioquímicos foram realizados pelo método enzimático no Laboratório de Nutrição do Hospital das Clínicas da Faculdade de Medicina de Ribeirão Preto da USP.

Foi utilizado o programa SPSS 14.0 para a análise estatística dos dados. A comparação entre as médias foi realizada por meio do teste $\mathrm{T}$ e a para a pesquisa de possível correlação entre as variáveis foi utilizado o coeficiente de correlação de Pearson. Assumiu-se $p<0,05$ como nível de significância.

\section{Resultados}

Houve grande variação das características clínicas e laboratoriais dentre os pacientes, como pode ser visto nas Tabelas 1, 2 e 3.

Não houve associação entre o colesterol total e a circunferência abdominal $(\mathrm{p}=0,88)$. Quando separamos pelo gênero verificamos que também não hou-

Tabela 1

Valor mínimo, valor máximo, média e desvio padrão das variáveis triglicérides, colesterol total, HDL, circunferência abdominal (CA) e circunferência do quadril (CQ).

\begin{tabular}{lccccc}
\hline & $\mathbf{N}^{\mathbf{0}}$ & Mínimo & Máximo & Média & Desvio padrão \\
\hline Triglicérides & 100 & 35 & 410 & 165,41 & 83,56 \\
Colesterol total & 100 & 119 & 431 & 239,95 & 70,76 \\
HDL colesterol & 100 & 29 & 93 & 53,4 & 13,90 \\
CA & 97 & 67 & 120 & 94,42 & 11,11 \\
CQ & 97 & 83 & 127 & 101,26 & 8,67 \\
\hline
\end{tabular}

\section{Tabela 2}

Valor mínimo, valor máximo, média e desvio padrão das variáveis triglicérides, colesterol total, HDL, circunferência abdominal (CA) e circunferência do quadril (CQ), nas mulheres

\begin{tabular}{lccccc}
\hline & n & Mínimo & Máximo & Média & Desvio padrão \\
\hline Triglicérides & 61 & 35 & 406 & 157,95 & 82,58 \\
Colesterol total & 61 & 119 & 414 & 240,95 & 66,34 \\
HDL colesterol & 61 & 33 & 86 & 55,75 & 13,31 \\
CA & 60 & 67 & 115 & 91,95 & 10,78 \\
CQ & 60 & 83 & 127 & 103,13 & 9,12 \\
\hline
\end{tabular}




\section{Tabela 3}

Valor mínimo, valor máximo, média e desvio padrão das variáveis triglicérides, colesterol total, HDL, circunferência abdominal (CA) e circunferência do quadril (CQ), nos homens

\begin{tabular}{lccccc}
\hline & n & Mínimo & Máximo & Média & Desvio padrão \\
\hline Triglicérides & 39 & 67 & 410 & 177,08 & 84,823 \\
Colesterol total & 39 & 138 & 431 & 238,38 & 78,044 \\
HDL colesterol & 39 & 29 & 93 & 49,72 & 14,164 \\
CA & 37 & 75 & 120 & 98,42 & 10,584 \\
CQ & 37 & 88 & 112 & 98,22 & 6,992 \\
\hline
\end{tabular}

ve associação ( $p=0,73$ e $p=0,60$ para homens e mulheres, respectivamente). A associação entre triglicérides e circunferência abdominal foi significativa ( $\mathrm{p}<0,0001$ ), mas ao separar por gêneros, houve associação entre triglicérides e circunferência abdominal nas mulheres $(\mathrm{p}=0,002)$ e não nos homens $(\mathrm{p}=0,07)$. Houve associação negativa entre o HDL e a circun- ferência abdominal $(\mathrm{p}=0,018)$, porém quando os gêneros foram analisados separadamente a associação entre HDL e circunferência abdominal não foi significativa $(\mathrm{p}=0,40$ e $\mathrm{p}=0,07$ para homens e mulheres, respectivamente). Estas associações estão representadas nos gráficos 1 e 2 .

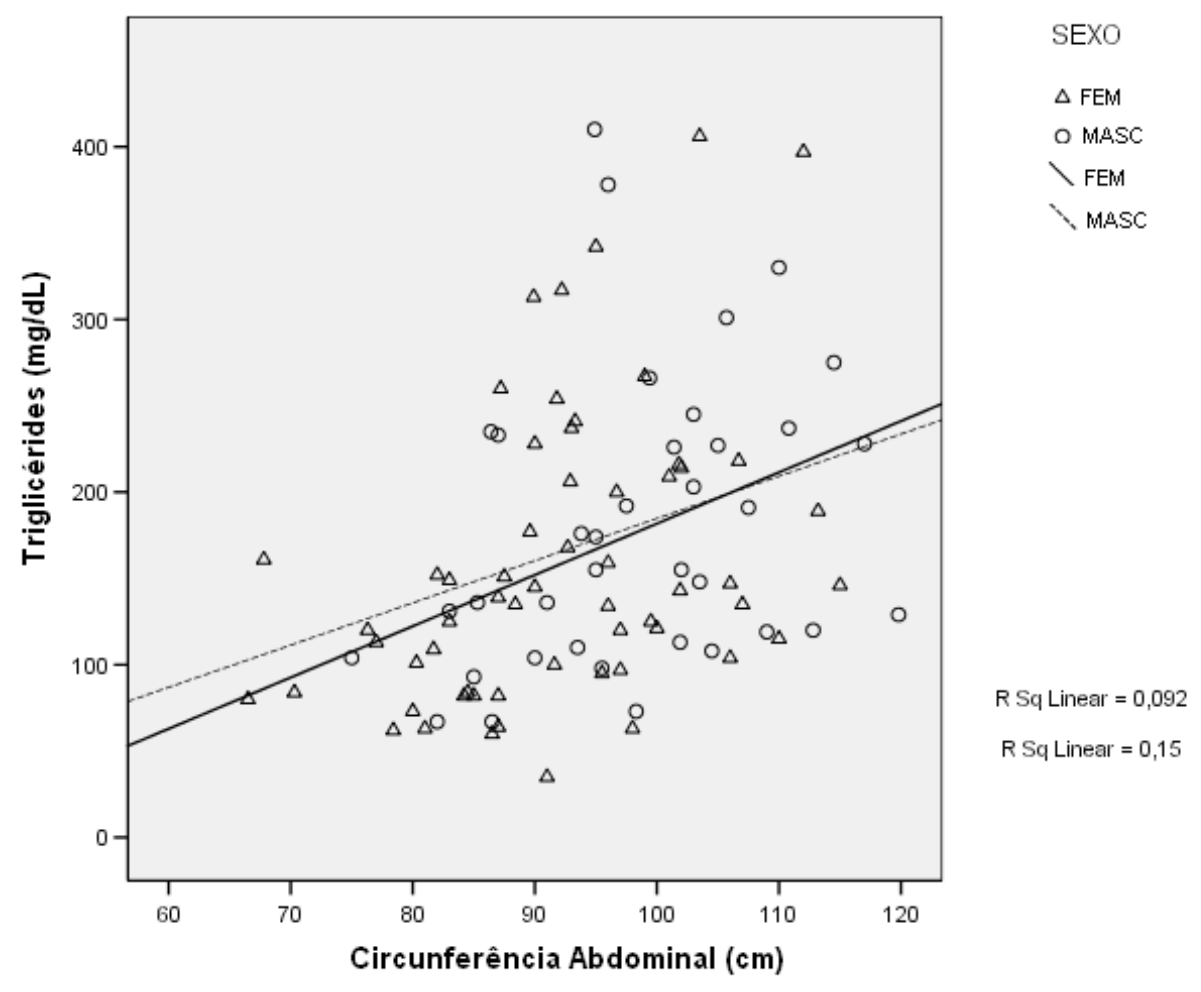

Gráfico 1 - Associação entre triglicérides e circunferência abdominal. 


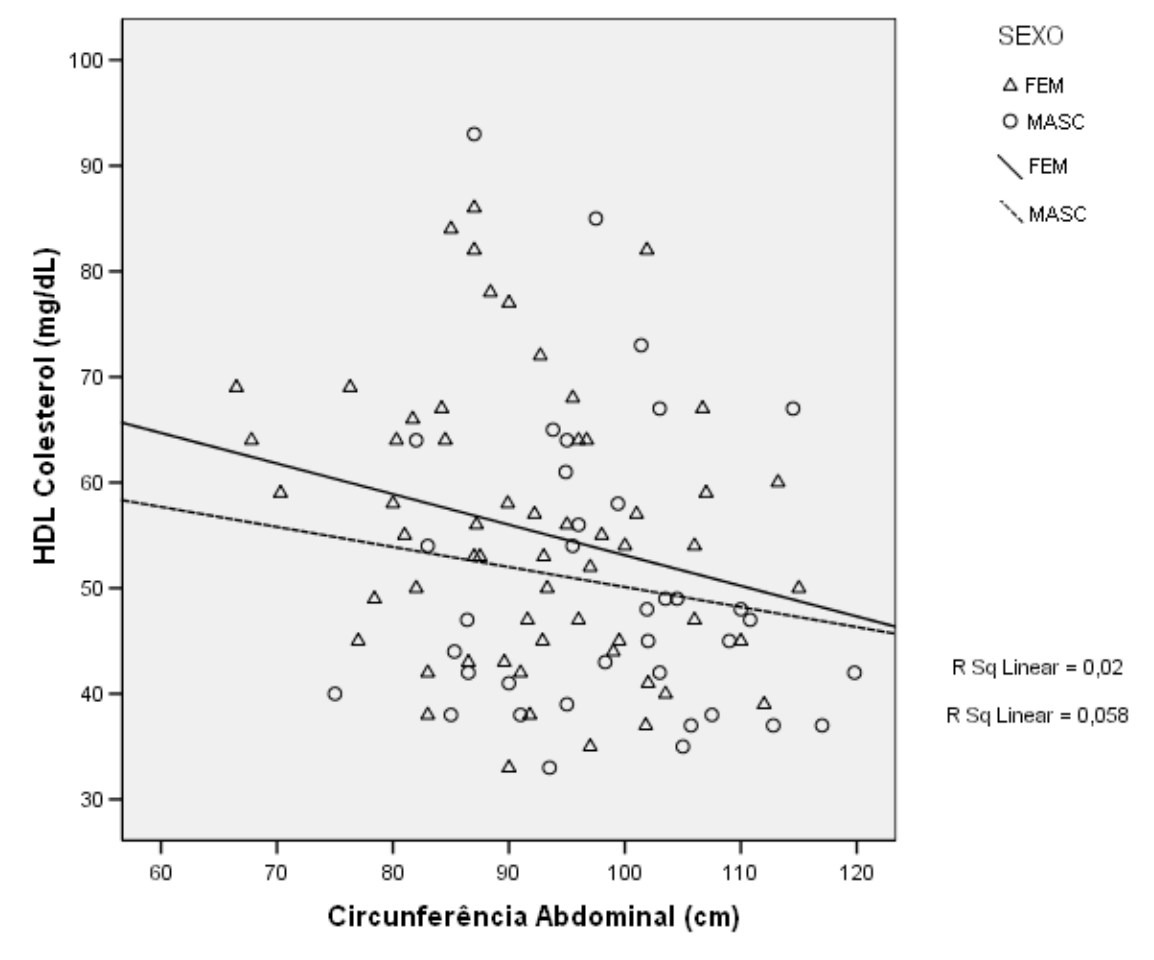

Gráfico 2 - Associação entre HDL e circunferência abdominal

\section{Discussão}

A obesidade tem recebido crescente importância pela sua alta incidência e prevalência e é fator de risco de inúmeras doenças, necessitando de maior atenção, principalmente nos idosos, pois estes têm menor sucesso no tratamento. A prevenção é a melhor forma de mudar esse quadro, e tem entre as opções os exercícios físicos, a dieta balanceada e fracionada e a interrupção do alcoolismo e do tabagismo.

A apresentação da obesidade é a que mais se relaciona a esses danos causados sobre a saúde, sendo que seu principal marcador é a gordura hepática (visceral). Como utilizada nesse estudo, a circunferência abdominal tem relação direta com o aumento da gordura visceral. ${ }^{15}$

Muitos estudos mostram que há grande relação entre obesidade e hipercolesterolemia, que causa muitas doenças, entre elas as coronariopatias. Valores elevados de colesterol total, triglicérides e LDL colesterol ocorrem em indivíduos com maior IMC, e também há menor nível de HDL colesterol nos obesos.

Drapeau e colaboradores realizaram estudo em mulheres no período pré e pós-menopausa com obesi- dade mórbida ou moderada. Observaram que a circunferência abdominal correlacionava-se inversamente ao HDL e razão entre HDL e colesterol total e diretamente com a glicemia, com maior intensidade nas mulheres com obesidade moderada do que naquelas com obesidade mórbida, antes da menopausa. Depois da menopausa, a circunferência abdominal não foi um bom indicador de anormalidades metabólicas em ambos os grupos. Não houve associação entre colesterol total e circunferência abdominal. ${ }^{16}$ Os resultados do presente estudo vão ao encontro dos resultados do estudo de Drapeau e colaboradores, uma vez que também não houve associação entre colesterol total e circunferência abdominal ( $\mathrm{p}=0,88)$, inclusive quando os gêneros foram analisados separadamente.

O colesterol total guarda estreita relação negativa com o aumento da idade ${ }^{17,18}$ e a justificativa para tal fato permanece sem etiologia única, aparentemente relacionada com mudanças na ingestão de nutrientes, peso corpóreo e estado de saúde. Estas informações suportam o fato de não ter sido encontrada associação, nesse estudo, entre circunferência abdominal e colesterol total. ${ }^{19}$

Em estudo realizado com 146 indivíduos saudáveis na sétima década de vida, Ostlund Jr e colabo- 
radores observaram associação entre os níveis de HDL e a circunferência abdominal $(\mathrm{p}<0,01) .{ }^{20}$ No presente estudo também foi visto que quanto maior a circunferência abdominal, menores eram os níveis de HDL colesterol, com associação significativa.

Ettinger, em 1992, estudou 4838 idosos e demonstrou associação do nível de triglicérides com a circunferência abdominal somente nas mulheres (2732). ${ }^{19}$ Neste estudo, não foi encontrada associação entre o nível de triglicérides e a circunferência abdominal no grupo estudado, porém quando foram separados pelo gênero os resultados vão ao encontro da associação já demonstrada por Ettinger, ou seja, somente no grupo das mulheres.

Apesar do envelhecimento e da natural e conhecida alteração da composição corporal que privilegia o aumento da gordura visceral o aumento da circunferência abdominal ainda guarda associação com o aumento do risco cardiovascular. A diminuição do HDL e o aumento dos triglicérides em idosos estão relacionados com o aumento da mortalidade cardiovascular. Com a associação encontrada, inclusive em idosos, entre a circunferência abdominal e o HDL colesterol e entre a circunferência abdominal e os triglicérides nas mulheres, entende-se que a utilização da circunferência abdominal para predizer risco cardiovascular permanece útil, especialmente pela grande facilidade da obtenção deste dado. Provavelmente, em idosos, o risco cardiovascular advindo da obesidade abdominal não tem na sua etiologia, exclusivamente, o aumento de lípides do sangue.

\section{Agradecimentos}

Financiamento: CNPq e FAEPA

\begin{abstract}
Model of the study: cross-sectional. Introduction: There are reports of an increase in the prevalence of dislipidemias with aging. It is necessary to determine the true impact of centripetal obesity on plasma levels lipids of older people. Objectives: To verify the possible association between the abdominal circumference, a marker of visceral fat, with the levels of blood lipids. Methods: The study included 98 patients with 60 to 79 years of age, residents in the western district of the city of Ribeirão Preto, 58 women. The mean age of the volunteers was 66.3 years. The following data were analyzed: abdominal circumference, hip circumference, triglycerides, total cholesterol and HDL cholesterol. The abdominal circumference was measured as a surrogate for centripetal obesity. Results: There was no association between total cholesterol and abdominal circumference $(p=0.88)$. There was also no association with different types of obesity ( $p=0.73$ and $p=0.60$ for men and women, respectively). The association between triglycerides and abdominal circumference was significant $(p<0.0001)$, but when obesity was classified according to types, there was association between triglycerides and abdominal circumference in women $(p=0.002)$ but not in men $(p=0.07)$. There was negative association between HDL cholesterol and abdominal circumference $(p=0.018)$, however when obesity types were analyzed separately the association between HDL and abdominal circumference was not significant ( $p=0.40$ and $p=0.07$ for men and women, respectively). Conclusion: Probably, in the old population, the cardiovascular risk resulting from centripetal obesity has not, in its etiology, the increase of lipids of the blood only.
\end{abstract}

Keywords: Cholesterol. Abdominal Circumference. Obesity. Triglycerides. Elderly.

\section{Referênclas bibllográficas}

1. Carvalho Filho ET. Fisiologia do envelhecimento. In: Papaléo Netto M. Gerontologia. São Paulo: Atheneu, 2002.

2. Instituto Brasileiro de Geografia e Estatística (IBGE). Diretoria de Pesquisas. Coordenação de População e Indicadores Sociais. Projeção da população do Brasil por sexo e por idade para o período 1980-2050. Disponível em: <http:// www.ibge.gov.br/home/estatistica/populacao/estimativa2005/metodologia.pdf>.
3. World Health Organization. Obesity: preventing and managing the global epidemic. Report of the WHO Consultation on Obesity. Geneva: World Health Organization; 1998.

4. Huang KC, Lee MS, Lee SD, Chang YH, Lin YC, Tu SH, et al.Obesity in the elderly and its relationship with cardiovascular risk factors in Taiwan. Obes Res. 2005;13:170-8.

5. National Institutes of Health. The sixth report of the Joint National Committee on prevention, detection, evaluation, and treatment of high blood pressure. National Institutes of Health, $\mathrm{NIH}$ Publication, 1997. 
6. Ran XW, Li XS, Tong NW, Li QF, Tang BD, Li XJ. Body fat distribution: its characteristics and relationship to cardiovascular risk factors in obese Chinese. Sichuan Da Xue Xue Bao Yi Xue Ban. 2004; 35:699-703.

7. Akahoshi M, Amasaki Y, Soda M, Tominaga T, Ichimaru S, Nakashima E, Seto S, Yano K. Correlation between fatty liver and coronary risk factors: a population study of elderly men and women in Nagasaki, Japan. Hypertens Res. 2001; 24:33743.

8. Windler E, Schöffauer M, Zyriax BC. The significance of low HDL-cholesterol levels in an ageing society at increased risk for cardiovascular disease. Diabetes Vasc Dis Res 2007; 4:136-142.

9. Caparevi Z, Kosti N. The influence of age and the beginning of menopause on the lipid status, LDL oxidation, and CRP in healthy women. Srp Arh Celok Lek. 2007;135:280-5.

10. Sonnichsen AC, Richter WO, Schwandt P . Body fat distribution and serum lipoproteins in relation to age and body weight. Clin Chim Acta. 1991; 202:133-40.

11. Niederauer CM, Binkley TL, Specker. Effect of truncal adiposity on plasma lipid and lipoprotein concentrations. J Nutr Health Aging. 2006; 10: 154-60.

12. Bouchard C. Atividade física e obesidade. In: Santarém JM. Treinamento de força e potência. São Paulo: Manole, 1993.

13. Halpern A, Matos AFG, Suplicy HI, Mancini MC, Zanella MT. Obesidade. São Paulo: Editora Lemos, 1998.
14. Cecil, Tratado de Medicina Interna, 2005, 22ª edição, Editora Elsevier, volume II, capítulo 233, pág 1557-8.

15. Farkas J, Angel A, Avigan MI. Studies on the compartmentation of lipid in adipose cells. II. Cholesterol accumulation and distribution in adipose tissue components. J Lipid Res. 1973; 14:344-56.

16. Drapeau V, Lemieux I, Richard D, Bergeron J, Tremblay A Biron S, Marceau P, Mauriège P. Waist circumference is useless to assess the prevalence of metabolic abnormalities in severely obese women. Obes Surg. 2007;17:905-9.

17. Clarke DA, Allen MF, Wilson FH: Longitudinal study of serum lipids: 12-Year report. Am J Clin Nutr 1967; 20:743-50.

18. Hershcopf RJ, Elahi D, Andres R, Baldwin H, Raizes GS, Schocken DD, Tobin JD: Longitudinal study in serum cholesterol in man: An epidemiologic search for an etiology. J Chronic Dis 1982; 35:101-14.

19. Ettinger WH, Wahl PW, Kuller LH, Bush TL, Tracy RP, Manolio TA, Borhani NO, Wong ND, O'Leary DH . Lipoprotein lipids in older people. Results from the Cardiovascular Health Study. The CHS Collaborative Research Group. Circulation1992; 86:858-69.

20. Ostlund RE Jr, Staten M, Kohrt WM, Schultz J, Malley M. The ratio of waist-to-hip circumference, plasma insulin level, and glucose intolerance as independent predictors of the HDL2 cholesterol level in older adults. N Engl J Med. 1990; 322:22934. 